\title{
Membrane protein CAR promotes hematopoietic regeneration upon stress
}

\section{Guojin Wu and Cheng Cheng Zhang}

Department of Physiology, University of Texas Southwestern Medical Center, Dallas, TX, USA

\section{ABSTRACT}

Haematologica 2021

Volume 106(8):2180-2190

\section{Correspondence:}

CHENGCHENG ZHANG

Alec.Zhang@UTSouthwestern.edu

Received: November 28, 2019.

Accepted: June 22, 2020.

Pre-published: June 25, 2020.

https://doi.org/10.3324/haematol.2019.243998

(C)2021 Ferrata Storti Foundation

Material published in Haematologica is covered by copyright. All rights are reserved to the Ferrata Storti Foundation. Use of published material is allowed under the following terms and conditions:

https://creativecommons.org/licenses/by-nc/4.0/legalcode. Copies of published material are allowed for personal or internal use. Sharing published material for non-commercial purposes is subject to the following conditions:

https://creativecommons.org/licenses/by-nc/4.0/legalcode, sect. 3. Reproducing and sharing published material for commercial purposes is not allowed without permission in writing from the publisher.
A dult hematopoietic stem cells (HSC) are quiescent most of the time, and how HSC switch from quiescence to proliferation following hematopoietic stress is unclear. Here we demonstrate that upon stress the coxsackievirus and adenovirus receptor CAR (also known as CXADR) is upregulated in HSC and critical for HSC entry into the cell cycle. Wild-type HSC were detected with more rapid repopulation ability than the CAR knockout counterparts. After fluorouracil treatment, CAR knockout HSC had lower levels of Notch1 expression and elevated protein level of Numb, a Notch antagonist. The Notch signaling inhibitor DAPT, dominant negative form of MAML (a transcriptional coactivator of Notch), or dominant negative mutant of LNX2 (an E3 ligase that acts on Numb and binds to CAR), all were capable of abrogating the function of CAR in HSC. We conclude that CAR activates Notch1 signaling by downregulating Numb protein expression to facilitate entry of quiescent HSC into the cell cycle during regeneration.

\section{Introduction}

In adults stem cells with self-renewal and differentiation capabilities are required for tissue homeostasis and regeneration. Quiescence protects stem cells from exhaustion. Hematopoietic stem cells (HSC) are largely quiescent during normal hematopoiesis, ${ }^{1}$ and adult hematopoiesis is sustained primarily by "shortterm" HSC (ST-HSC). ${ }^{1}$ During injury or inflammation, quiescent HSC enter the cell cycle to accelerate hematopoietic flux, ${ }^{1.3}$ and cycling HSC return to quiescence after the injury is repaired or inflammation is resolved. Multiple signaling pathways are known to be important for regulation of cell fates and regeneration of HSC..$^{4-10}$ The molecular mechanisms that regulate HSC to transit from quiescence to proliferation during regeneration remain largely unknown.

The coxsackievirus and adenovirus receptor (CAR, also known as CXADR) was first reported to mediate viral attachment and infection ${ }^{11}$ and later was demonstrated to be a tight junction protein. ${ }^{12}$ CAR expression is required for normal atrioventricular conduction and cardiac function. ${ }^{13}$ Its constitutive expression in various cancerous and normal tissues has also been reported..$^{14,15}$ CAR was also reported to be critical for survival of oral squamous cell carcinomas ${ }^{16}$ Interestingly, CAR expression increases during tissue regeneration, ${ }^{17}$ suggesting that it plays an important role in repairing injury. Here we demonstrate that CAR expression is transiently increased in HSC during fluorouracil (5-FU)-induced hematopoietic injury and bone marrow (BM) transplantation and supports HSC regeneration. CAR does not alter HSC self-renewal but rather induces quiescent HSC to enter cell cycle. Mechanistic studies indicated that CAR activates Notch1 signaling in stressed HSC by degrading the Notch inhibitor Numb.

\section{Methods}

\section{Mice}

$\mathrm{CAR}^{\text {LoxPloxp }}$ mice and UBC-Cre-ERT2 mice were purchased from Jackson Laboratory. Mice were maintained at the University of Texas Southwestern Medical Center animal facility. All animal experiments were performed with the approval of The University of Texas Southwestern Committee on Animal Care. 


\section{Competitive reconstitution analysis}

CD45.2 donor bone marrow cells $\left(3 \times 10^{5}\right.$ unless otherwise indicated) were mixed with an equal number of freshly isolated CD45.1 competitor BM cells, and the mixture was injected intravenously via the retro-orbital route into 6- to 9-week-old CD45.1 mice that had been previously irradiated with a total dose of $10 \mathrm{~Gy}$, essentially as we described. ${ }^{18-20} \mathrm{BM}$ cells $\left(1 \times 10^{6}\right)$ collected from primary recipients were used for the secondary transplantation. In order to measure reconstitution of transplanted mice, peripheral blood was collected at the indicated times after transplantation and the presence of CD $45.1^{+}$and CD $45.2^{+}$ cells in lymphoid and myeloid compartments was measured.

\section{5 -fluorouracil treatment}

5-FU was administered intraperitoneally to female wild-type (WT) or CAR conditional knockout (cKO) mice at a dose of 150, 250 , or $300 \mathrm{mg} / \mathrm{kg}$. In order to test Notch signaling, DAPT (Selleckchem, cat. \#S2215) was injected at $10 \mathrm{mg} / \mathrm{kg}$. Differences in survival of the two groups were analyzed using a log-rank test.

\section{Retrovirus infection}

$L N X 1$ and $L N X 2$ were cloned from cDNA of mouse intestine. The dominant negative forms (DN-LNX1 and DN-LNX2) were sub cloned ${ }^{21}$ and retroviral plasmids MSCV-DN-LNX1-IRESGFP and MSCV-DN-LNX2-IRES-GFP were constructed using standard methods. The retroviral plasmids with PCL-ECO (2:1) were transfected using PolyJet (SignaGen) into $293 \mathrm{~T}$ cells. The resulting retroviral supernatant was collected $48-72$ hours later and used for infection. At 6 days after 5-FU injection, the Lin cells from the $\mathrm{BM}$ of $C A R \mathrm{cKO}$ mice were isolated, and the Lin cells were resuspended in viral supernatants $\left(1 \times 10^{5}\right.$ cells $\left./ \mathrm{mL}\right)$ with $4 \mu \mathrm{g} / \mathrm{mL}$ polybrene and centrifuged at $2,000 \mathrm{rpm}$ for 2 hours before culturing for 24 hours in StemSpan (StemCell Technologies) in the presence of $10 \mathrm{ng} / \mathrm{mL}$ stem cell factor, 20 $\mathrm{ng} / \mathrm{mL}$ thrombopoietin, and $10 \mathrm{ng} / \mathrm{mL}$ interleukin 3. Cells were then resuspended in viral supernatant for another round of infection. After 24 hours, green fluorescent protein positive $\left(\mathrm{GFP}^{+}\right)$ cells were isolated for repopulation assay.

\section{Statistics}

Statistical significance of differences was assessed using 2tailed Student's t-test. Animal survival analysis was assessed with long-rank test. A $P$-value of 0.05 or less was considered significant. Values are reported as mean \pm standard error of the mean.

\section{Results}

\section{CAR expression is elevated in stressed hematopoietic stem cells}

We compared CAR mRNA levels in HSC, hematopoietic progenitors, and differentiated hematopoietic cells before and after stress. As reported previously, ${ }^{22} C A R$ is expressed in differentiated cells and not stem cells or hematopoietic progenitors with the exception of granulocyte-monocyte progenitor (GMP) cells (Figure 1A). Shortly after treatment of mice with 5-FU or after BM transplantation, CAR expression was greatly enhanced in phenotypical LT-HSC (identified as Lin-Sca1 ${ }^{+} \mathrm{cKit}^{+} \mathrm{CD} 135$ CD34 or LSKFC) compared to untreated mice (Figure 1B). CAR expression returned to the original low level at the later time point (Figure 1B). However, ST-HSC, multipotent hematopoietic progenitors (MPP) and hematopoietic progenitors did not increase CAR expression after 5-FU treatment (Online Supplementary Figure 1). In order to prove CAR is expressed on plasma membrane of HSC, we stained BM cells from 5-FU treated mice with anti-mCAR. Real-time qantitative polymerase chain reaction (RT-qPCR) and CAR cKO BM cells both prove the specific binding of the anti-mCAR (Online Supplementary Figure 2; Figure 1B). The surface CAR expression was detected in LSK population after 5-FU treatment (Figure 1C). Competitive repopulation assay with sorted CAR negative and positive expressed LSKFC cells from 5-FU treated mice indicates that both subpopulations had similar repopulation abilities (Figure 1D and E; Online Supplementary Figure 3). These results imply that CAR plays a role in HSC during regeneration induced by stress.

\section{CAR supports hematopoietic regeneration after injury}

$C A R$ deletion in mice is embryonically lethal.$^{23}$ In order to study the function of CAR, CAR ${ }^{\text {loxplloxp }}$ mice were crossbred with UBC-CreERT2 mice. In the resulting mice, global knockout (KO) of CAR can be induced with tamoxifen treatment; these mice are thereafter referred to as $C A R$ conditional $\mathrm{KO}(\mathrm{cKO})$ mice. The hematopoietic system is damaged by treatment with the chemotherapy drug 5-FU. ${ }^{24}$ CAR cKO mice were more sensitive to 5-FU treatment and died faster. After administration of 300 $\mathrm{mg} / \mathrm{kg} 5$-FU, $60 \%$ of $C A R$ cKO mice died within 2 weeks, whereas all WT mice (which were UBCCreERT2/CAR ${ }^{\text {wtwt }}$ mice with tamoxifen treatment) survived (Figure 2A). This indicates that CAR is important in the response to injury of the hematopoietic system. In order to compare kinetics of hematopoietic system recovery in WT and cKO mice, we treated mice with 250 $\mathrm{mg} / \mathrm{kg} 5$-FU, a dose that does not cause death of the WT mice. The complete blood count $(\mathrm{CBC})$ data indicated CAR cKO mice had a slower recovery, although there was not a significant difference in hematopoietic cell counts between WT and CAR cKO mice before 5-FU treatment. On day 8 after 5 -FU treatment, total white blood cell (WBC) and individual lineage cell numbers (including neutrophils, monocytes, eosinophils, basophils, and lymphocytes) had decreased relative to pretreatment levels. Numbers rebounded more rapidly in WT mice than in the CAR cKO mice. On day 14 post 5FU treatment there were an average of $12.8 \times 10^{3} \pm 1.5$ WBC per $\mu \mathrm{L}$ in WT mice and only $6.2 \times 10^{3} \pm 0.4 \mathrm{WBC}$ per $\mu \mathrm{l}$ in cKO mice (Figure 2B). On day 17 post treatment, the $C A R$ cKO mice and WT mice had equivalent WBC counts of about $12 \times 10^{3}$ per $\mu \mathrm{L}$. A significant difference in neutrophil numbers between WT and CAR cKO mice was detected on day 12 (Figure 2B). Red blood cell (RBC) numbers decreased more slowly than WBC counts and the lowest cell counts were detected on day 14 in both WT and CAR cKO mice. Although there were no significant differences detected before day 14, the WT mice had significant more RBC than cKO mice in peripheral blood between day 14 and 17 (Figure 2B). Specifically, Mac1 ${ }^{+}$ cells and $\mathrm{B}_{2} 20^{+}$cells had significantly faster recovery in WT mice than in CAR cKO mice after 5-FU treatment (Online Supplementary Figure 4). These results indicate that $C A R$ cKO mice produce hematopoietic blood cells more slowly after 5-FU treatment, which explains why mice lacking CAR die after a dose of 5-FU that is sub-lethal dose to WT mice. 


\section{CAR stimulates progenitor production during regeneration}

The Lin-Sca1 ${ }^{+} \mathrm{cKit}^{+}$(LSK) population of hematopoietic cells is primarily responsible for production of progenitors during regeneration. ${ }^{25}$ We compared the production of hematopoietic progenitors by WT and CAR cKO mice after dosing with $150 \mathrm{mg} / \mathrm{kg} 5$-FU and after BM transplantation (Figure $3 \mathrm{~A}$ ). The CAR cKO mice produced progenitors more slowly during recovery. Before 5-FU administration there were no differences in pre- $\mathrm{B}$ or myeloid colony forming units (CFU) in WT and CAR cKO mice. Four days after 5-FU injection, there were significantly more pre-B CFU and myeloid CFU but fewer granulocyte, erythrocyte, monocyte, megakaryocyte (GEMM) CFU in WT mice than in CAR cKO mice, suggesting a slower hematopoietic differentiation in CAR cKO mice upon 5FU-induced injury. When the mice recovered from injury at 7 days after 5-FU injection, the CAR cKO mice had the same number of myeloid CFU and the difference in pre$\mathrm{B}$ cell CFU between WT and CAR cKO mice was smaller than on day 4 (Figure 3A). We also injected WT or CAR
cKO BM cells into lethally irradiated recipient mice and conducted CFU assays for the BM cells. WT donor cells produced more pre- $\mathrm{B}$ and myeloid $\mathrm{CFU}$ than $\mathrm{CAR} \mathrm{CKO}$ donor cells on days 9 and 11 post transplantation (Figure 3B). The difference disappeared at 2 months after transplantation, suggesting CAR cKO only took effect during regeneration. These results indicate that CAR stimulates production of differentiated progenitors.

\section{CAR stimulates entry of "long-term"-hematopoietic stem cells into cell cycle}

Although CAR promotes progenitor production during regeneration, it did not affect frequency of progenitors or stem cells during homeostasis (Online Supplementary Figure 5). During regeneration, HSC enter the cell cycle and expand. ${ }^{2}$ During expansion, HSC may self-renew or differentiate. In order to investigate whether CAR regulates the function of LT-HSC, ST-HSC, and MPP upon transplantation stress, we collected WT and cKO LT-HSC (LSKFC) and ST-HSC plus MPP (Lin-Sca1 $\left.{ }^{+} \mathrm{cKit}^{+} \mathrm{CD} 34^{+}\right)$for competitive repopulation assays. From day 17 after trans-
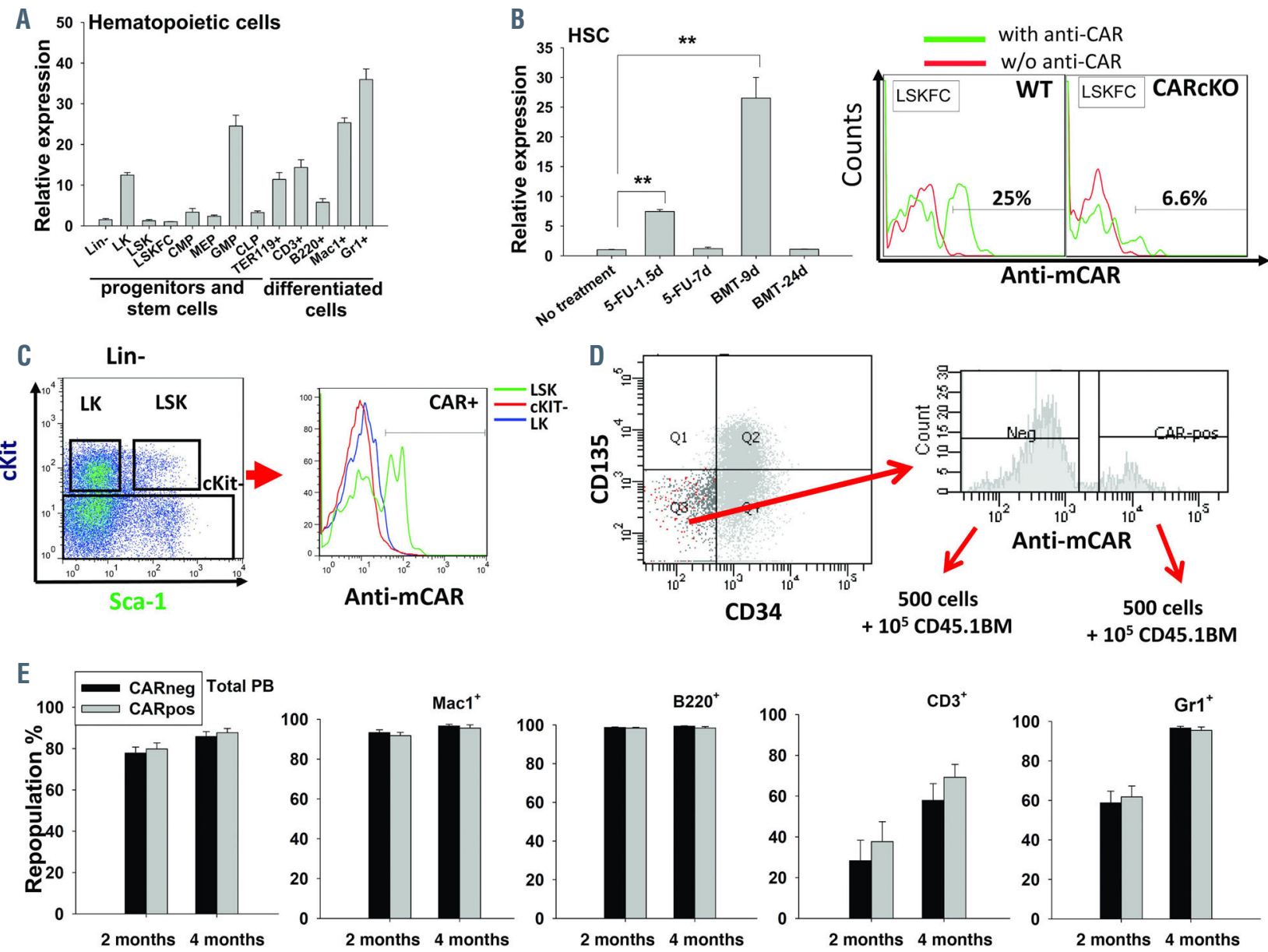

Figure 1. CAR is expressed in differentiated hematopoietic cells, and its expression increases in hematopoietic stem cells during regeneration. (A) CAR expression (determined by real-time qantitative polymerase chain reaction [RT-qPCR]) in hematopoietic cells, hematopoietic stem cells (HSC), and progenitors including Lin-, LK (Lin-cKit+Sca1-), LSK (Lin-cKit ${ }^{+} S c a 1^{+}$), LSKFC (Lin-Sca1 ${ }^{+}$cKit $^{+}$CD135 CD34), CMP (LKCD34 ${ }^{+}$FcrR ${ }^{-}$), MEP (LKCD34 FcrR), granulocyte-monocyte progenitor (GMP) $\left(\mathrm{LKCD} 34^{+} \mathrm{FcrR}{ }^{+}\right)$, and CLP $\left(\mathrm{Lin}-\mathrm{CK} \mathrm{Kit}^{\text {low }} \mathrm{Sca} 1^{\text {low }} \mathrm{CD} 135^{+} \mathrm{CD} 127^{+}\right)$. (B) Right panel, CAR expression (determined by RT-qPCR) in LT-HSC (selected as LSKFC) at day 1.5 and day 7 after 5-fluorouracil (5-FU) treatment (5-FU-1.5d and 5-FU-7d, respectively) and at 9 and 24 days post bone marrow (BM) transplantation (BMT-9d and BMT24d, respectively); Left panel, BM cells from wild-type (WT) and CAR conditional knockout (cKO) for analysis CAR expression on LSKFC. (C) CAR surface expression on LK, LSK and Lin-cKit- population after 5-FU treatment. (D) Flow chart of repopulation experiment for LSKFC CAR- or CAR+. (E) Repopulation results of LSKFC CAR or $\mathrm{CAR}^{+}(n=7-9)$. One and half days after treated with 5-FU, BM cells from mice were isolated for flow cytometry analysis (panel B (left) and C) or for isolating LSKFC cells (D and E). The mRNA levels were calculated based on non-treated LSKFC, and experiments were repeated three times. $* * P<0.001$. 


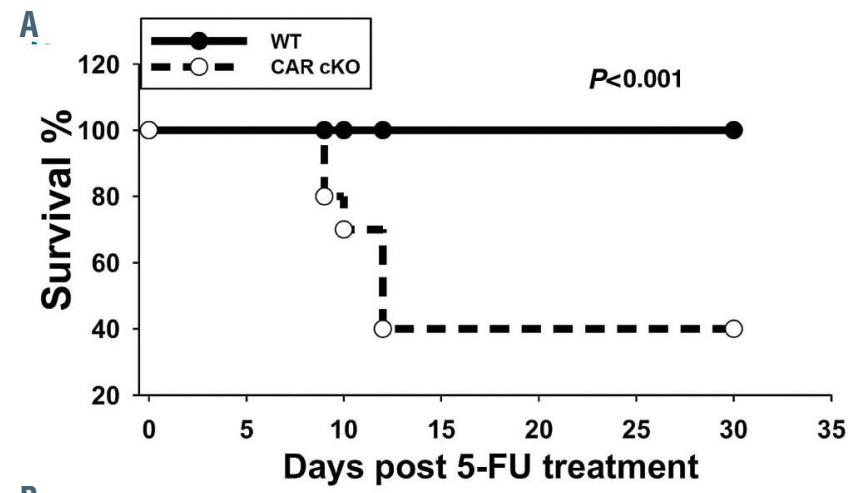

Figure 2. CAR enhances recovery from 5-fluorouacil treatment. (A) Survival curves of wild-type (WT) $(n=6)$ and $C A R$ conditional knockout (cKO) $(\mathrm{n}=10)$ mice treated with 300 $\mathrm{mg} / \mathrm{kg} 5$-fluorouracil (5-FU). The mice administered with tamoxifen 1 month before 5-FU treatment. (B) One month after tamoxifen treatment, WT $(n=6)$ and CAR cKO $(n=8)$ mice were treated with $250 \mathrm{mg} / \mathrm{kg} 5$-FU, and the complete blood counts at the indicated days were evaluated. ${ }^{*} P<0.05$ $* * P<0.001$.

B
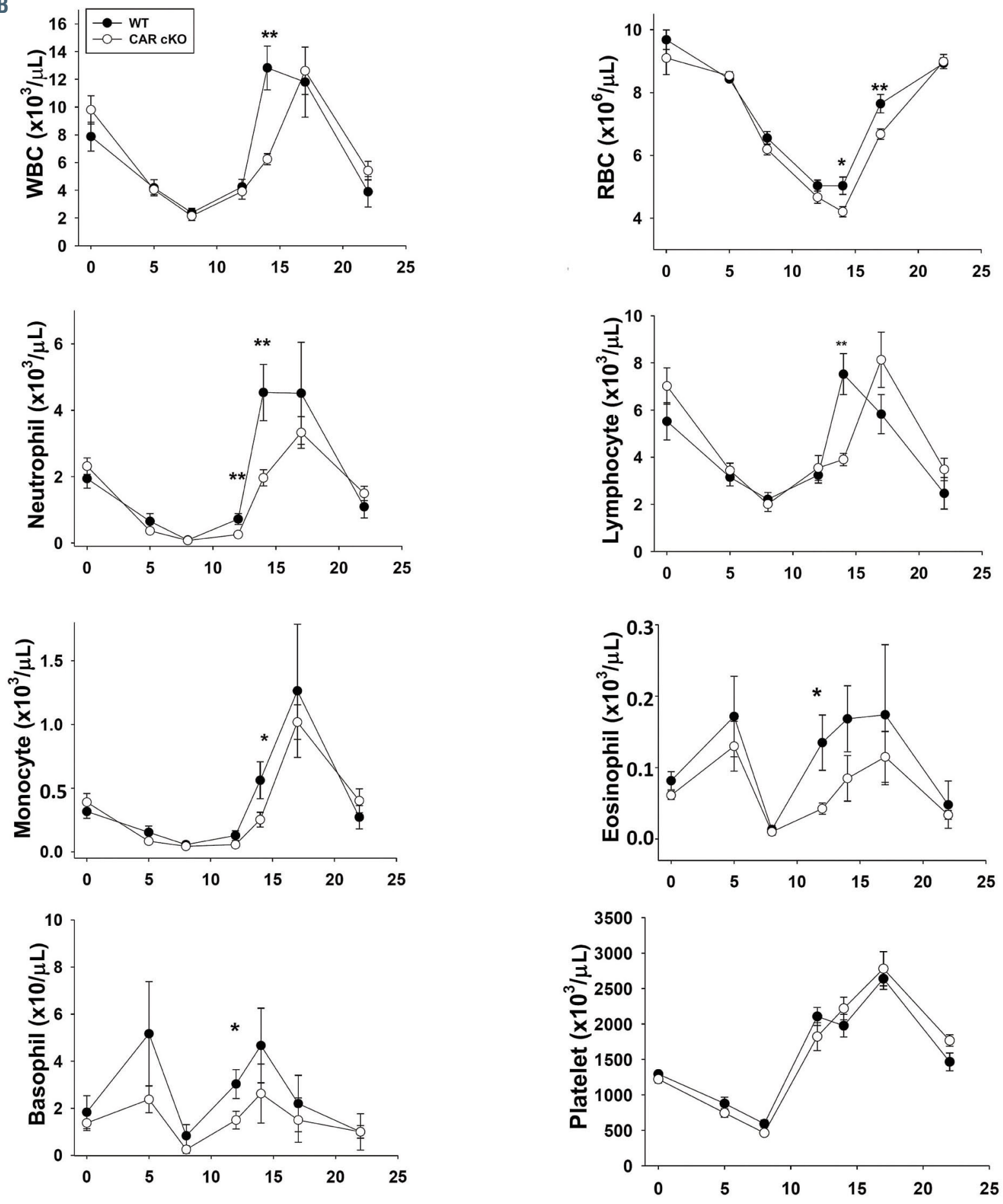

Days post 5-FU treatment 
plantation, the repopulation percentage of WT LT-HSC donors gradually increased to above $35 \%$, whereas the percentage decreased to below $2 \%$ for WT ST-HSC plus MPP (Figure 4A). This indicated that LT-HSC play the major role in regeneration and that ST-HSC and MPP are quickly exhausted.

When WT and CAR cKO donors were compared, WT LT-HSC repopulated about twice as efficiently as CAR cKO cells in the first 6 weeks (Figure 4A). There was only a small difference for ST-HSC plus MPP between WT and CAR cKO cells on days 21 and 23 in Mac1 ${ }^{+}$population. In the $\mathrm{B} 220^{+}$population there were significant differences in LT-HSC, but not in ST-HSC plus MPP, between WT and CAR cKO donors (Figure 4A). This suggests that CAR cKO impairs the function of LT-HSC but not of ST-HSC and MPP. At 170 days after transplantation, the repopulation percentages donor LSKFC of WT and CAR CKO LTHSC groups did not differ significantly (Figure 4B), indicating that CAR deficiency does not alter LT-HSC activity during regeneration. These results indicate that CAR stimulates regeneration mainly by affecting LT-HSC.

In order to evaluate whether lack of CAR is detrimental after bone marrow transplantation, we conducted another repopulation assay (Figure 4C). Here WT or CAR cKO BM cells were mixed with an equal number of CD45.1 BM cells and were injected into lethally irradiated recipient mice. WT donor cells showed greater repopulation ability in both myeloid and lymphoid cells than CAR cKO donor cells in first 6 weeks after transplantation. After 6 weeks, the difference between WT and CAR cKO repopulation gradually diminished. At 16 weeks after BM transplantation, the repopulation percentage of the donor
BM cells from CAR cKO mice was not significantly different from that in mice that received WT cells (Figure 4C). In the second transplantation, CAR cKO donor cells had no defects in long-term repopulation ability (Figure 4C). This serial transplantation analysis indicates that the selfrenewal of LT-HSC was not affected by CAR. In addition, CAR did not affect homing ability of HSC (Online Supplementary Figure 6). The limiting dilution analysis indicates that $C A R$ cKO did not change the frequency of HSC in BM (Online Supplementary Table1). This result suggests that CAR enhances the speed of hematopoietic repopulation after BM transplantation but does not change HSC activity over the long term. Together, these results demonstrate that CAR supports hematopoietic regeneration after stress. Furthermore, the donor BM cells from Scl-CreERT/CARloxp/loxp mice in which CAR was specifically $\mathrm{cKO}$ in hematopoietic cells also had defects in initial repopulation after BM transplantation (Online Supplementary Figure 7), indicating that CAR on the hematopoietic cells plays a major role in regeneration. In repopulation assay with $C A R \mathrm{cKO}$ recipient mice, $C A R$ cKO in the donor hematopoietic cells still resulted in defects in repopulation (Online Supplementary Figure 8), suggesting that CAR in the BM microenvironment is not essential for the function of CAR in regeneration.

Next, we assessed phenotypical LT-HSC (LSKFC), stem cells, and multiple progenitors in WT and CAR cKO mice after 5-FU treatment. None of these populations altered once the mice recovered from 5-FU injury (Figure 4D). In order to test whether the functional LT-HSC were affected by CAR after injury, BM transplantation was conducted with the donor cells collected from mice 1 month after 5 -
A
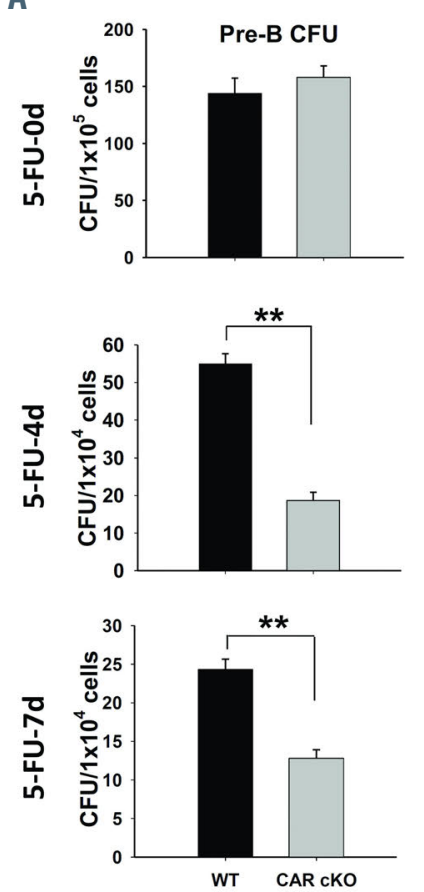
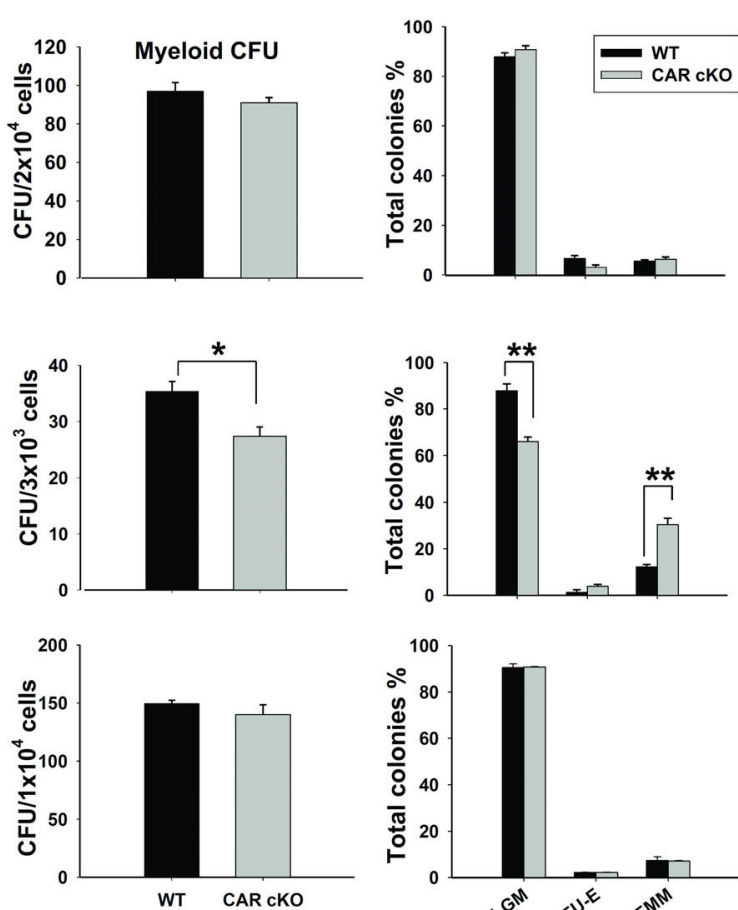

B
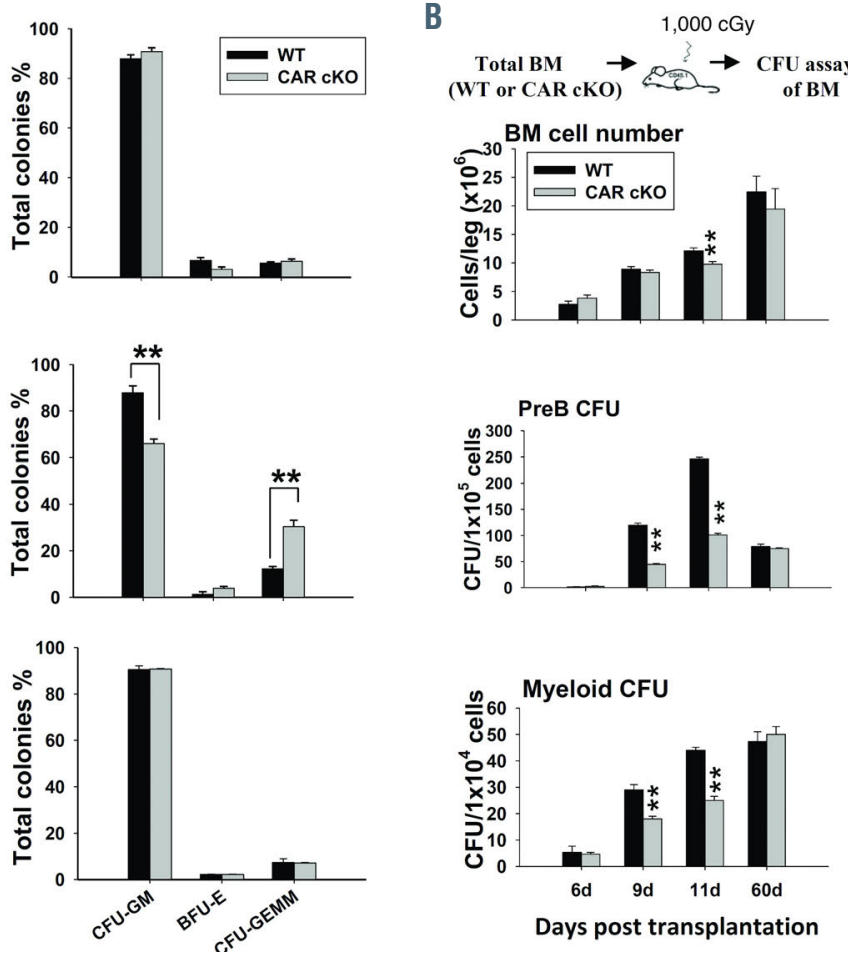
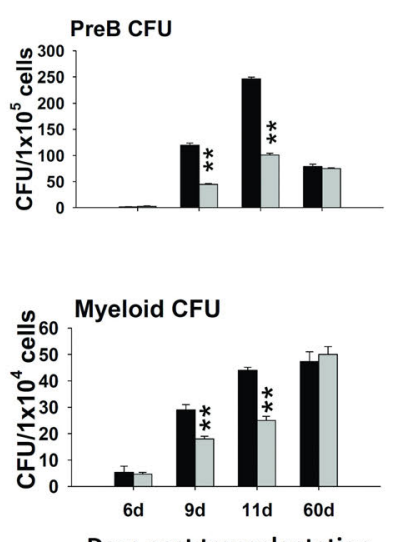

Days post transplantation

Figure 3. CAR stimulates progenitor production after 5-fluorouacil treatment and bone marrow transplantation. (A) One month after tamoxifen treatment, bone marrow (BM) cells from wild-type (WT) or CAR conditional knockout (cKO) mice were isolated before and at indicated days after 5-fluorouracil (5-FU) treatment (150 $\mathrm{mg} / \mathrm{kg}$ ) for colony forming units assays. (B) CFU assays of BM cells after transplantation of $1 \times 10^{6}$ total $B M$ cells into lethally irradiated WT. $* P<0.05$; $\star * P<0.001$. 
FU treatment. There was no difference in the long-term repopulation ability between WT and CAR cKO donors in this experiment (Figure 4E). Together, our results indicated that self-renewal of LT-HSC was not affected by CAR neither during homeostasis nor during regeneration.

In order to further understand how CAR affects LT-HSC, the population LSKFC cells were identified from mice on different days after 5 -FU treatment and cell cycle states were analyzed (Figure 5). Before 5-FU injection, there was no difference between WT and CAR cKO cell cycle status. At 1.5 days after treatment, there was a significantly lower frequency of WT LSKFC in the G0 stage, and more WT LSKFC cells in apoptosis than prior to treatment because 5FU kills cycling cells. After 3 days, more WT LSKFC than
CAR cKO cells were in G1 stage and fewer were in G0 stage (Figure 5). These results suggest that CAR is needed for the transition into the cell cycle. On day 5 post 5 -FU treatment, more than $90 \%$ of LSKFC cells were cycling and there were no differences between WT and CAR cKO populations. This implies that CAR is needed immediately after injury. Compared to CAR cKO mice, LSKFC of WT mice returned to homeostasis earlier, as more WT than CAR cKO LSKFC were at the G0 stage on day 6. In addition, $\mathrm{CAR}^{+}$LSKFC cells were positive for Ki67 staining and thus were cycling, whereas most CAR- LSKFC cells were Ki67 negative right after 5-FU treatment (Online Supplementary Figure 9). Thus, CAR appears to stimulate quiescent HSC to enter the cell cycle.
A

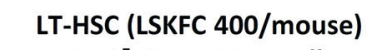
$+5 \times 10^{5}$ CD45. 1 BM cells

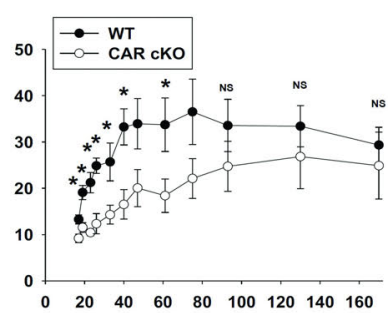

\section{No}

Total PB
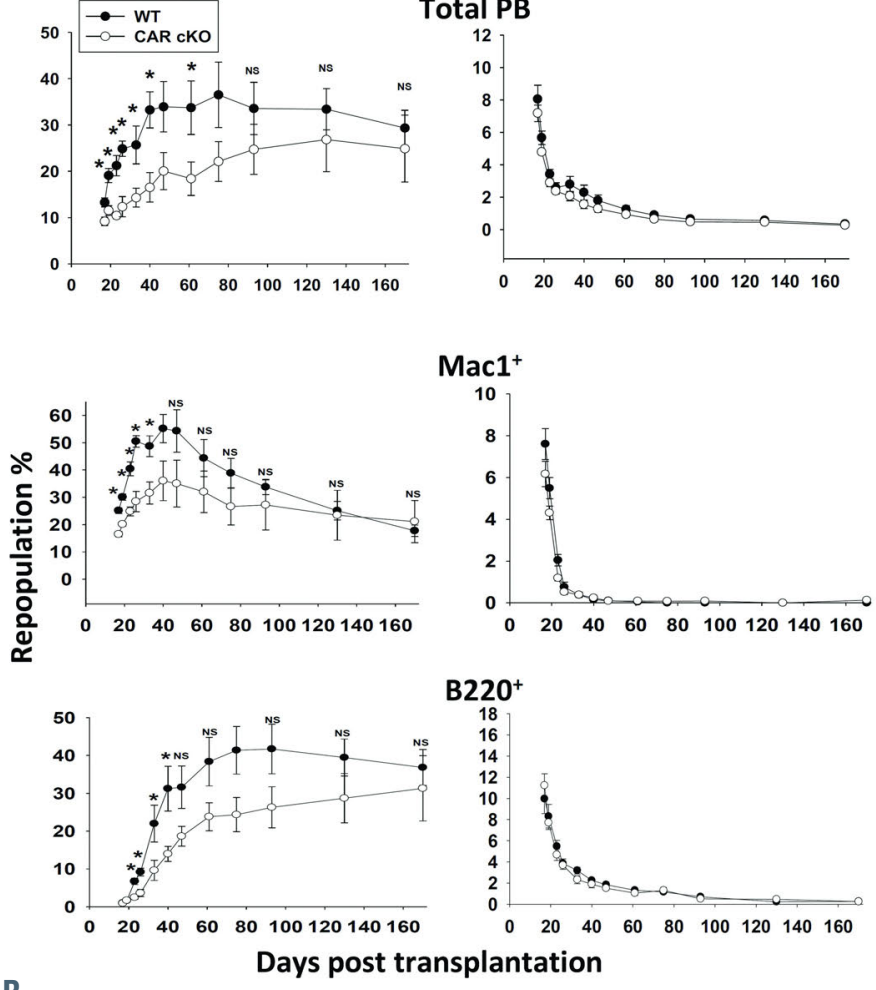

B

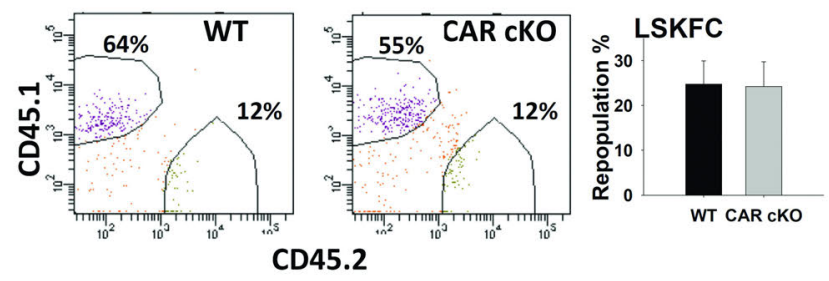

C
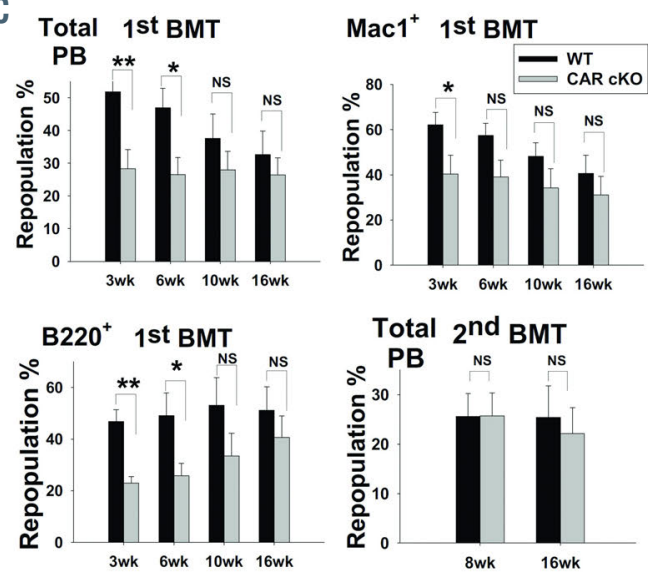

D
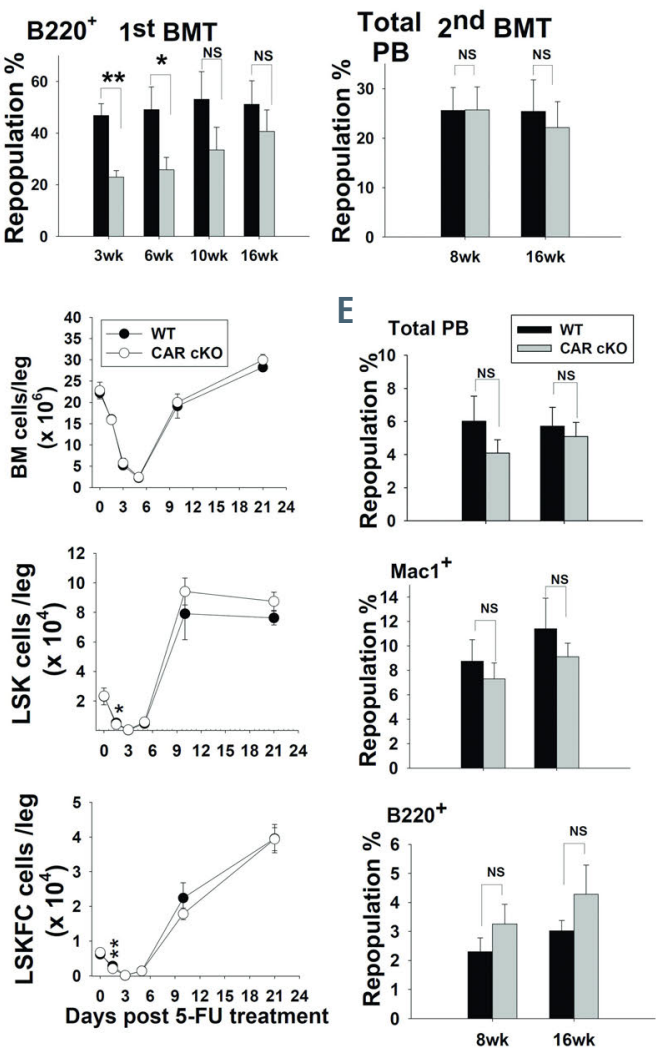

Figure 4. CAR stimulates regeneration by forcing hematopoietic stem cells into the cell cycle but does not affects self-renewal. (A) Competitive repopulation assays were performed with LT-HSC (LSKFC, LSKCD34-CD135) or "short-term" HSC (ST-HSC) plus multipotent hematopoietic progenitors (MPP) (LSKCD34") from wild-type (WT) or CAR conditional knockout (cKO) donors (1 month after tamoxifen treatment) and CD45.1 WT competitor bone marrow (BM). Percentages of donor peripheral leukocytes in total peripheral blood (PB), the Mac1 $1^{+}$population, and the B220+ population after BM transplantation are plotted. (B) Percentages of donor LT-HSC (CD45.2) in total BM LT-HSC (CD45.1 plus CD45.2) 170 days after transplantation. (C) Competitive repopulation assays after initial BM transplantation were performed with a 1:1 ratio of donor (CD45.2) and CD45.1 WT competitor BM. Shown are the percentages of donor peripheral leukocytes (CD45.2) in total PB, the Mac1 ${ }^{+}$ population, and the B220+ population. Data are means \pm standard error of the mean, $n=7-9$ mice. (D) Number of LSK and LSKFC cells in BM before and after treatment with $150 \mathrm{mg} / \mathrm{kg}$ 5-FU ( $\mathrm{n}=3-9$ mice per group). (E) Donor mice (1 month after tamoxifen treatment) were injected with $150 \mathrm{mg} / \mathrm{kg} 5$-FU, and $1 \mathrm{month}$ later, competitive repopulation assays were performed with a 1:1 ratio of donor and CD45.1 WT competitor BM. Shown are the percentages of donor peripheral leukocytes in total $\mathrm{PB}$, the Mac1 $1^{+}$population, and the $\mathrm{B} 22 \mathrm{O}^{+}$population. Data are means \pm standard error of the mean, $\mathrm{n}=9-10$ mice. ${ }^{\star} P<0.05 ; * \star P<0.001$. NS: no significant difference. 


\section{CAR stimulates Notch signaling}

We sought to identify the underlying mechanism by which CAR mediates hematopoietic regeneration after stress. CAR binds to LNX, an E3 ligand of Numb, ${ }^{21}$ suggesting the function of CAR may be related to Numb. After 5-FU treatment, Numb protein levels in HSC gradually decreased, and the population with no Numb staining increased from $5.7 \pm 0.3 \%$ on day 0 to $62.4 \pm 1.4 \%$ on day 5 (Figure $6 \mathrm{~A}$ ). On day 7, the Numb-negative population decreased to $6.8 \pm 1.5 \%$ (Figure $6 \mathrm{~A}$ ) as HSC had returned to homeostasis. Concordantly, the Numb mRNA levels decreased by more than 16 times on day 5 relative to pre-treatment levels and had returned to pretreatment levels on day 7 (Figure 6A). HSC in WT mice entered the cell cycle earlier than did CAR cKO counterparts after 5-FU treatment (Figure 5). At 1.5 days after 5FU treatment, there were fewer LSKFC cells that did not stain for Numb in CAR cKO mice than WT mice (Figure $6 \mathrm{~B})$, whereas there were no differences detected before or 5 days later after 5-FU treatment (Online Supplementary Figure 10A). The Numb mRNA levels in CAR cKO mice were similar to those in WT mice at 1.5 days after 5-FU treatment (Online Supplementary Figure 10B), suggesting that CAR mediates the degradation of Numb protein but does not affect Numb mRNA. In addition, there was fewer Numb protein in CAR ${ }^{+}$LSKFC cells than in CAR LSKFC cells (Figure 6C). Because Numb specifically inhibits Notch signaling, ${ }^{26-28}$ these results suggest that Notch signaling is involved in CAR-mediated hematopoietic regeneration.

Notch signaling was previously reported to play an important role in hematopoietic regeneration. ${ }^{6,29,30}$ In order to test how Notch signaling influences the function of CAR during regeneration, we treated mice with DAPT, an inhibitor of Notch signaling, with or without $300 \mathrm{mg} / \mathrm{kg} 5$-FU. Whereas 5-FU treatment decreased survival of CAR cKO mice relative to WT counterparts, there was no difference in survival between 5-FU-treated WT and CAR cKO mice when Notch signaling was blocked by DAPT (Figure 6D). This implies that CAR stimulates regeneration via Notch signaling. At 1.5 days after 5-FU treatment, $4.2 \%$ of LSKFC cells were Notch1 positive in CAR cKO mice compared to $8.7 \%$ in WT mice, whereas $27.7 \%$ and $27 \%$ of LSKFC cells in CAR cKO and WT mice were positive for Notch2, respectively (Figure 6E). This indicate that Notch1, but not Notch2, is involved in the function of CAR. There were significantly lower levels of hes1, hey1, and myc, all Notch1 target genes, in CAR cKO mice compared to WT mice at 1.5 days after 5-FU treatment; there were no differences in levels of these mRNA prior to 5-FU treatment (Figure 6F). In addition, CAR cKO resulted in a defect in T-cell repopulation (Online Supplementary Figure 11), which further suggests a connection between CAR and Notch1, as Notch1 affects T-cell development. ${ }^{31}$ Notch1 target genes indeed increased right after 5-FU treatment (Online Supplementary Figure 12). With 5-FU treatment, CAR+ HSC had more surface Notch1 protein than CAR- HSC (Figure 6G). Overexpression of CAR in LSKFC cells in vitro also increased Notch1 target gene expression (Online Supplementary Figure 13). In order to further confirm that CAR stimulates Notch signaling, we overexpressed the dominant negative MAML (dnMAML) ${ }^{32-34}$ in 5-FU treated Lin- BM cells from UBC-Cre-ERT2/CARloxp/loxp mice
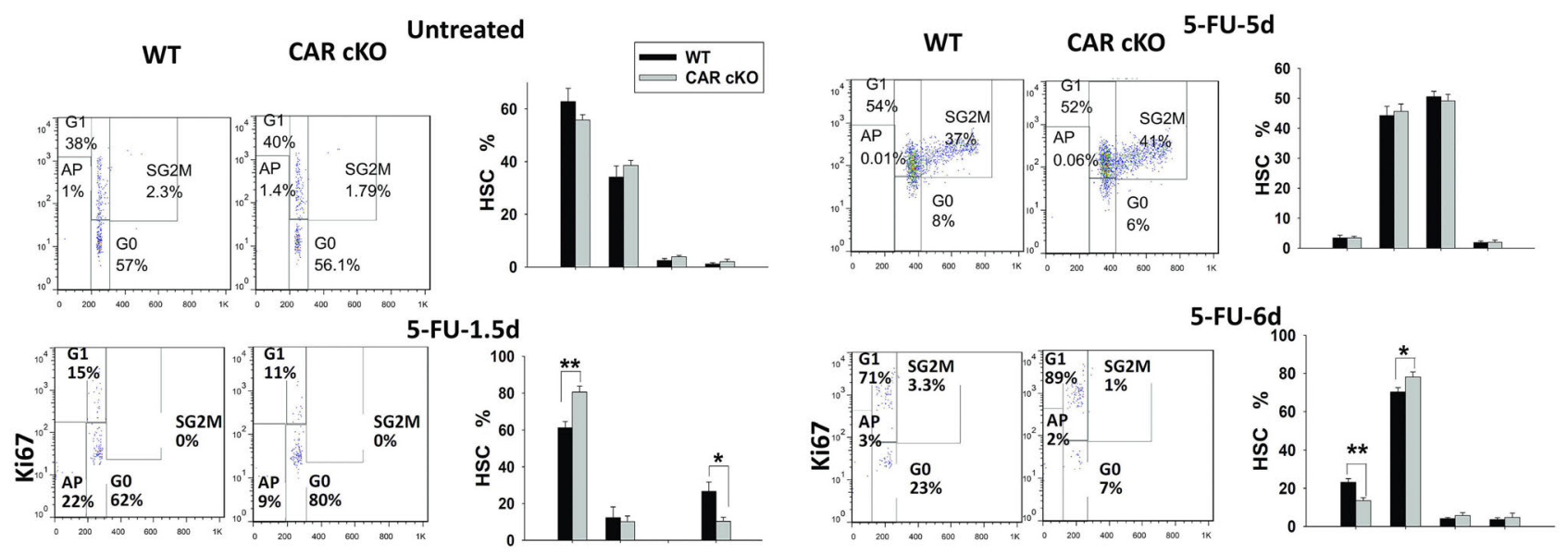

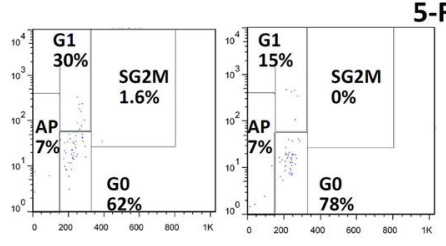

Hoechst
5-FU-3d

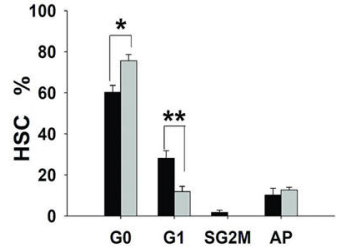

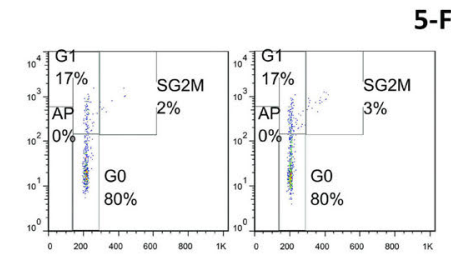

Hoechst
5-FU-7d

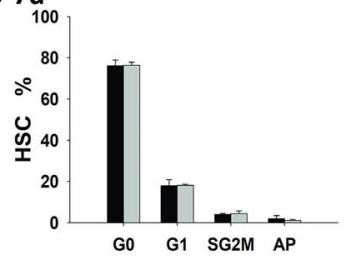

Figure 5. CAR stimulates hematopoietic stem cells proliferation after 5-fluorouracil treatment. Before and after 5-fluorouracil (5-FU) (150 mg/kg) treatment, cell cycle states of LSKFC cells were determined. The flow cytometry images are the representative results of bone marrow (BM) samples from wild-type (WT) or CAR conditional knockout ( $\mathrm{CKO}$ ) mice different times after 5-FU treatment, and the summaries of each time point after 5-FU treatment are on the right of flow cytometry images. The percentages of cell cycle states in total hematopoietic stem cells (HSC) (LSKFC) are indicated. Every groups include $n=4-9$ mice. G0: cell in G0 phase; G1; SG2M: cell in S, G2 and M phase; AP: cells in apoptosis. $* P<0.05, * * P<0.001$. 
A
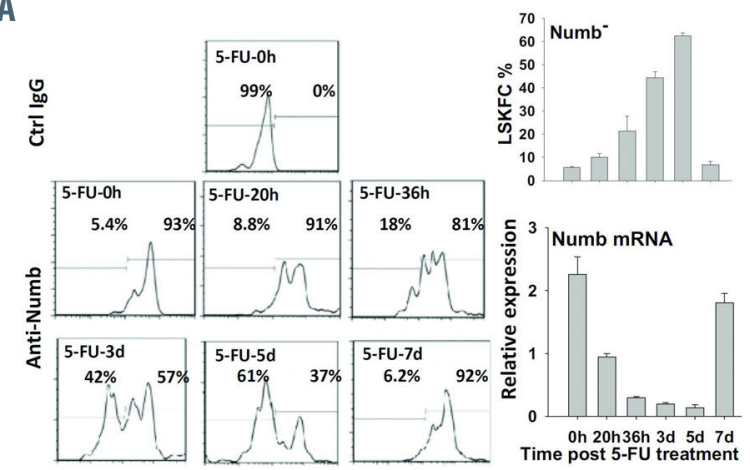

E
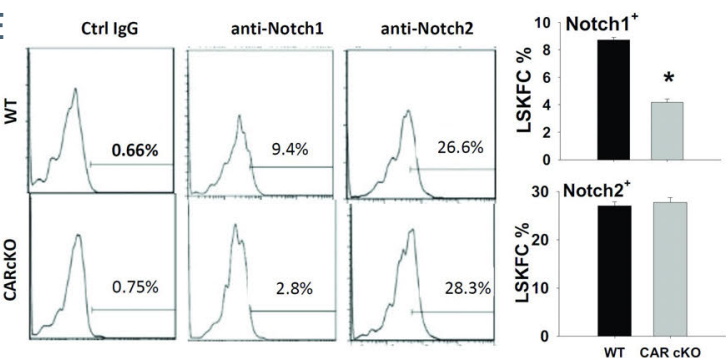

$\mathrm{H}$
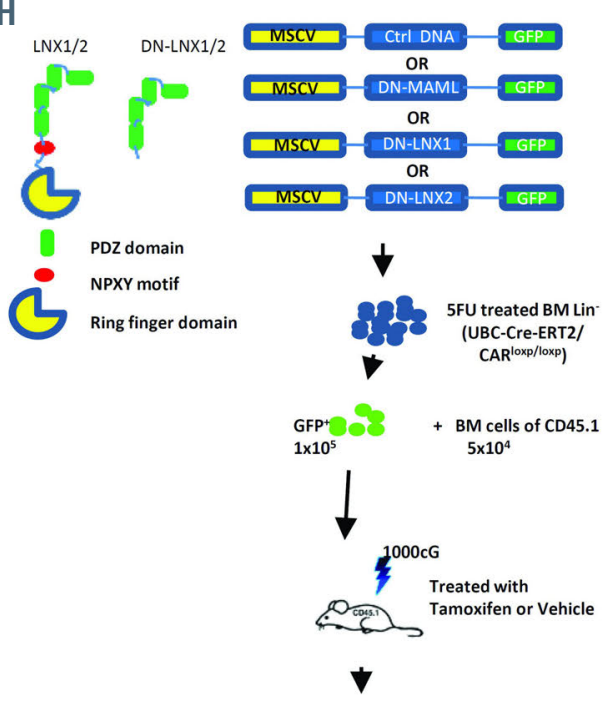

Analysis of PB from 17d
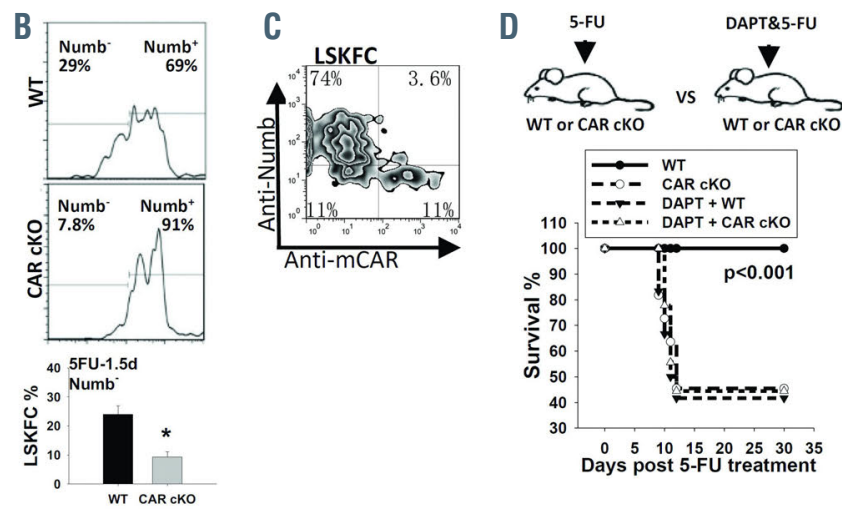

$\mathrm{F}$
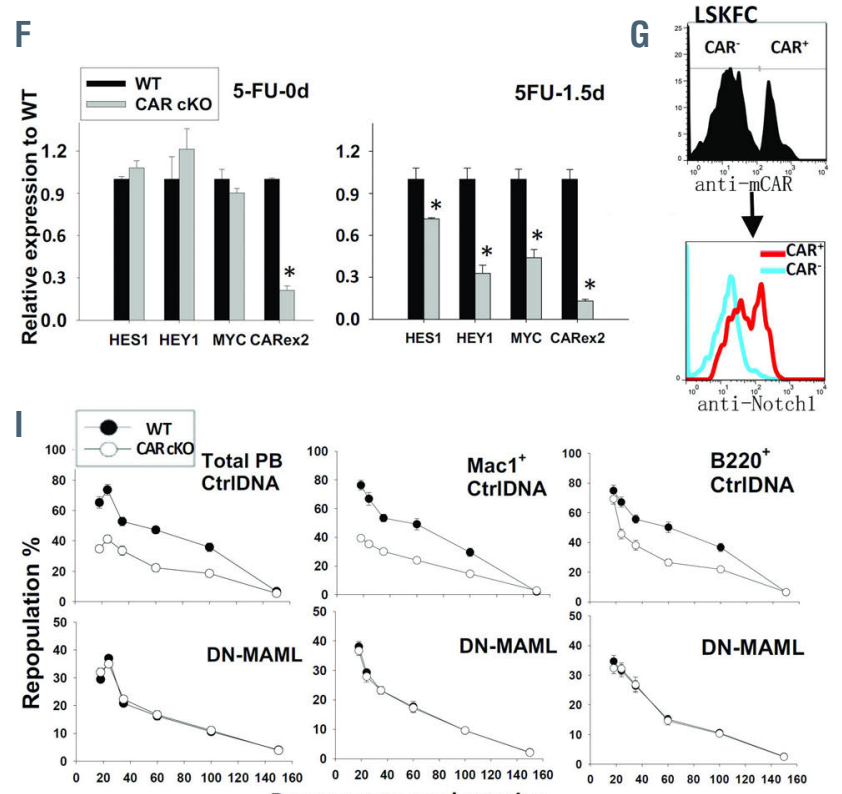

Days post transplantation

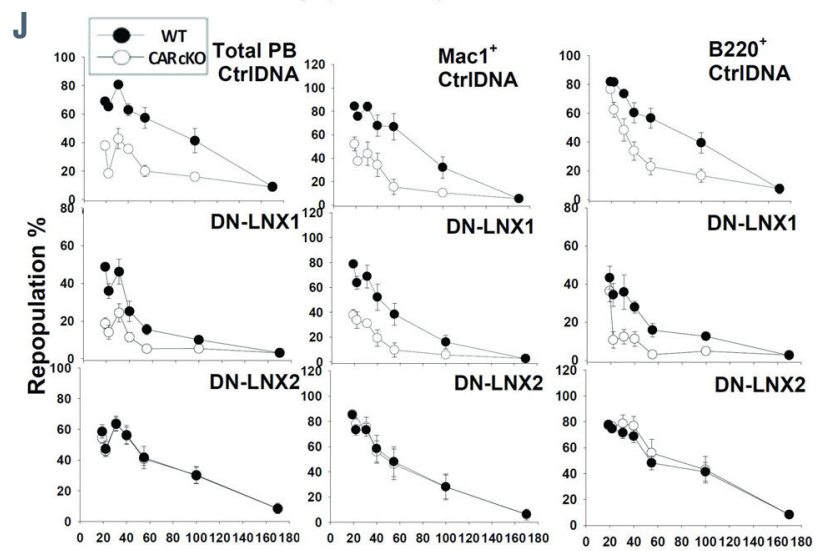

Days post transplantation

Figure 6. CAR enhances regeneration by stimulating Notch signaling. (A) Left panel: representative flow cytometry analyses of Numb negative and positive staining of LSKFC populations of 5-fluorouracil (5-FU) treated mice. Right top panel: Quantification of Numb negative staining in LSKFC. Right bottom panel: Quantification of Numb mRNA levels in LSKFC populations of 5-FU treated mice (the mRNA levels were calculated based on the 20-hour group, and experiments were repeated three times). (B) Top panel: representative flow cytometry analyses of Numb negative and positive staining in LSKFC populations at 36 hours after 5 -FU treatment. Bottom panel: quantification of percentage of Numb negative LSKF cells in wild-type (WT) and CAR conditional knockout (cKO) cells, $n=5$ mice per group. (C) Numb and mouse CAR co-staining in LSKFC population of mice at 36 hours after 5-FU treatment. (D) Survival curves of WT and CAR cKO mice after injection with 300 $\mathrm{mg} / \mathrm{kg}$ 5-FU and Notch signal inhibit DAPT, $\mathrm{n}=9-12$ mice per group. (E) Right panel: representative flow cytometry analyses of membrane Notch1/2 staining in LSKFC populations at 36 hours after 5-FU treatment. Left top panel: quantification of percentage of Notch1-stained cells in LSKFC populations. Left bottom panel: quantification of percentage of Notoch2-stained cells in LSKFC populations, $\mathrm{n}=9$ mice per group. (F) mRNA levels of CAR Notch target genes hes1, hey1, and myc in LSKFC populations before (right panel) and 36 hours after 5-FU treatment (left panel). (G) Notch1 and mCAR co-staining in LSKFC population of mice at 36 hours after 5 FU treatment. (H) Flow chart of experiment used to test whether Notch, LNX1 or LNX2 is involved in CAR function. DN-MAML, DN-LNX1 or DN-LNX2 was expressed in Lin- BM cells from CAR CKO mice without treatment of tamoxifen (UBC-Cre-ERT2/CAR ${ }^{\text {loxp/loxp }}$ ), and these cells were used in repopulation assays. The recipient mice were treated with tamoxifen as CAR cKO group and treated with vehicle as WT group. (I and J) Percent repopulation of peripheral blood (PB) (left panels), the Mac1 population (middle panels), and the $\mathrm{B}_{2} 20^{+}$population (right panels) by cells, $\mathrm{n}=8-10$ mice per group. ${ }^{\star} P<0.05$. 
(CAR cKO mice before treatment with tamoxifen) to specifically inhibit Notch signaling (Figure $6 \mathrm{H}$ ). In the competitive repopulation assay, the recipient CD45.1 mice were treated with tamoxifen to induce $C A R \mathrm{cKO}$ in the donor cells, with vehicle treatment as controls. With overexpressed dnMAML, the donor WT and CAR cKO $B M$ cells showed the same repopulation abilities, whereas WT BM cells had a stronger repopulation ability than CAR cKO BM cells in the control condition (Figure 6I). In order to test whether enhanced Notch1 signaling rescues the phenotype of CAR deficiency, CAR cKO mice injected with 5 -FU $(300 \mathrm{mg} / \mathrm{kg})$ were treated with valproic acid, a Notch1 activator. ${ }^{35}$ These mice survived significantly longer than $C A R$ cKO mice without valproic acid treatment (Online Supplementary Figure 14). In addition, valproic acid restored the repopulation ability of CAR cKO HSC (Online Supplementary Figure 15).

LNX contains a PDZ domain that may bind to the PDZ binding motif in the intracellular domain of CAR. ${ }^{36,37}$ There are two forms of LNX: LNX1 and LNX2. ${ }^{36,37} \mathrm{In}$ order to test the roles of LNX1 and LNX2 in the function of CAR, the dominant negative (DN) forms of the LNX proteins (DN-LNX1 and DN-LNX2) with the ring finger domains and the NPXY motifs deleted ${ }^{21}$ were over expressed in 5-FU treated Lin- BM cells from UBC-Cre-

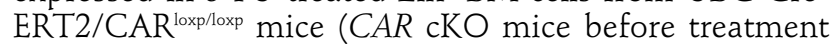
with tamoxifen), and a competitive repopulation assay was performed (Figure $6 \mathrm{H}$ ). The cells expressing control DNA had significantly weaker repopulation ability in CAR cKO mice than in WT mice in both myeloid $\left(\mathrm{Mac1}^{+}\right)$and lymphoid (B220+) populations (Figure 6J). Repopulation of WT and CAR cKO mice was similar when donor cells expressed DN-LNX2, while donor cells expressing DN-LNX1 less effectively repopulated CAR cKO mice (Figure 6J). At 170 days after transplantation, repopulation percentages were similar in all groups (Figure 6J), indicating that lack of CAR had no effect on the total HSC pool. In order to further explore the relationship among CAR, LNX2 and Numb, we overexpressed CAR, LNX2 or DN-LNX2 in Lin- cells and evaluated the protein levels of Numb in LSK cells with flow cytometry. Overexpression of CAR or LNX2 decreased the Numb level in LSK cells, whereas DN-LNX2 elevated Numb expression (Online Supplementary Figure 16). These data suggest that LNX2, but not LNX1, is involved in the function of CAR (Online Supplementary Figure 17).

\section{Discussion}

Substantial efforts have been dedicated toward uncovering the mechanisms that regulate HSC niche maintenance." ${ }^{8}$ Recent studies demonstrated the essential roles of BM endothelial cells and osteolineage cells in regulating HSC regeneration following myelotoxicity ${ }^{33.40}$ and identified two BM endothelial cell-derived paracrine factors, PTN and EGF, and osteolineage cell-derived Dkk1 as regulators of HSC regeneration in vivo. ${ }^{38,41,42}$ Here we identified CAR as a membrane protein on HSC that is rapidly upregulated after stress, whose transient expression induces quiescent HSC to enter the cell cycle to counteract the hematopoietic injury. Without CAR, the process was delayed, and the mice in which CAR was conditionally deleted were more sensitive to injury than WT mice. Lack of CAR neither changed the pool nor altered the self-renewal of HSC.
Mechanistically we showed that elevated CAR upon stress activates Notch1 signaling by down regulating expression of Notch antagonist Numb to facilitate entry of quiescent HSC into the cell cycle. To our knowledge, this is the first report demonstrating the role of CAR in HSC regeneration.

The Notch pathway plays an important part in many developmental processes and appears to regulate many adult stem cell fate decisions. ${ }^{43,44}$ While it was reported that Notch signaling is dispensable for HSC self-renewal and maintenance during hematopoiesis, ${ }^{45}, 46$ Notch1 can promote the expansion of LT-HSC while preserving selfrenewal ability. ${ }^{47,48}$ The expression of the canonical Notch ligands Jagged-1 and Jagged-2 by endothelial cells support hematopoietic regeneration. ${ }^{10,29}$ Varnum-Finney et al. showed that Notch2 enhances the rate of formation of short-term repopulating multi-potential progenitor cells as well as LT-HSC after 5-FU treatment. ${ }^{6}$ Our results indicate that expression of CAR in quiescent LT-HSC increases Notch1 expression and induces HSC to enter the cell cycle immediately after injury and has no effect on HSC that have entered the cell cycle. Of note, this Notch1-mediated proliferation upon stress does not alter self-renewal of HSC.

Numb is a membrane-associated, evolutionarily conserved adaptor protein that regulates cell fate determination via its ability to antagonize Notch signaling. ${ }^{49,50}$ Normal HSC differentiation and self-renewal occur in the absence of Numb during homeostasis. 51 We observed that Numb expression decreased after injury and then returned to normal levels after the injury was repaired (Figure 6A), and CAR accelerated Numb protein degradation before Numb mRNA levels decreased (Figure 6B). A low level of Numb protein might be necessary for functional Notch signaling during regeneration. Low Numb mRNA levels during regeneration may be due to inhibition by Notch signaling. ${ }^{52}$ LNX proteins are E3 ligases that act on Numb ${ }^{53}$ and that may bind to the PDZ motif of CAR. ${ }^{36,37}$ Here we demonstrated that CAR expressed on HSC activates Notch signaling via LNX2, suggesting the PDZ motif of CAR plays a critical role in hematopoietic regeneration. CAR and CAR can form trans-interaction with a low affinity, ${ }^{54}$ and this transinteraction may stimulate CAR downstream signaling. However, in CAR cKO recipient mice, CAR expressed on donor hematopoietic cells still plays a critical role in regeneration in repopulation assay (Online supplementary Figure 8). Analysis of BM cells after 5-FU injection, HSC were more prone to increase CAR expression compared to other cells (Figure 1C). These results indicate that CAR expressed in the BM microenvironment is not important in HSC regeneration, and an unknown ligand may act on CAR and on HSC to regulate transition from quiescence to proliferation during hematopoietic stress. These possibilities warrant future investigations.

How HSC detect signals sent upon injury remains unknown. Low oxygen tension (hypoxia) is thought to be a characteristic of the quiescent HSC niche ${ }^{55}$ and oxygen levels may change markedly after radiation and chemotherapy. ${ }^{56}$ CAR expression is repressed by hypoxia inducible factor-1a (Hif-1a). ${ }^{57}$ We therefore propose that the oxygen increase resulting from hematopoietic injury may suppress Hif-1a expression, leading to induction of CAR expression. This transient elevation of CAR levels activates Notch1 signaling by down regulating expression of Notch antagonist Numb. Notch signaling could promote expansion of HSC by preventing hematopoietic cell differentiation upon 
stress. ${ }^{10,29,47,48}$ Our study suggests that the coupling of Notch with CAR enables a checked cell fate of HSC with increased cell cycling but not over-differentiation. Overall the mechanism facilitates the entry of quiescent HSC into the cell cycle with maintenance of stemness. Our continuous study in this subject may lead to development of novel strategies for promotion of stem cell regeneration.

\section{Disclosures}

No conflicts of interest to disclose.

\section{Contributions}

$G W$ contributed to design, experimental performance, interpretation, and writing; $C C Z$ contributed to design and writing.

\section{Acknowledgments}

We thank the NIH (1R01CA172268), the Leukemia \& Lymphoma Society (1024-14), the March of Dimes Foundation (1-FY14-201), the Cancer Prevention and Research Institute of Texas (RP180435), and the Robert A. Welch Foundation (I1834) for generous support.

\section{References}

1. Busch K, Klapproth K, Barile M, et al. Fundamental properties of unperturbed haematopoiesis from stem cells in vivo. Nature. 2015; 518(7540):542-546.

2.Wilson A, Laurenti E, Oser G, et al. Hematopoietic stem cells reversibly switch from dormancy to self-renewal during homeostasis and repair. Cell. 2008;135(6): 1118-1129.

3. Essers MA, Offner S, Blanco-Bose WE, et al. IFN $\alpha$ activates dormant haematopoietic stem cells in vivo. Nature. 2009;458(7240): 904-908.

4. Goessling W, North TE, Loewer S, et al. Genetic interaction of PGE2 and Wnt signaling regulates developmental specification of stem cells and regeneration. Cell. 2009;136(6):1136-1147.

5. Seita J, Weissman IL. Hematopoietic stem cell: self-renewal versus differentiation. Wiley Interdiscip Rev Syst Biol Med. 2010;2(6):640-653.

6. Varnum-Finney B, Halasz LM, Sun M, Gridley T, Radtke F, Bernstein ID. Notch2 governs the rate of generation of mouse long-and short-term repopulating stem cells. J Clin Invest. 2011;121(3):1207-1216.

7. Mendelson A, Frenette PS. Hematopoietic stem cell niche maintenance during homeostasis and regeneration. Nat Med. 2014;20(8):833-846.

8. Morrison SJ, Scadden DT. The bone marrow niche for haematopoietic stem cells. Nature. 2014;505(7483):327-334.

9. Zhang CC, Sadek HA. Hypoxia and metabolic properties of hematopoietic stem cells. Antioxid Redox Signal. 2014;20(12):18911901.

10. Guo P, Poulos MG, Palikuqi B, et al. Endothelial jagged-2 sustains hematopoietic stem and progenitor reconstitution after myelosuppression. J Clin Invest. 2017; 127(12):4242-4256

11. Bergelson JM, Cunningham JA, Droguett G, et al. Isolation of a common receptor for Coxsackie $B$ viruses and adenoviruses 2 and 5. Science. 1997;275(5304):1320-1323

12. Cohen CJ, Shieh JT, Pickles RJ, Okegawa T, Hsieh J-T, Bergelson JM. The coxsackievirus and adenovirus receptor is a transmembrane component of the tight junction. Proc Natl Acad Sci U S A. 2001;98(26):15191-15196.

13. Lim B-K, Xiong D, Dorner A, et al. Coxsackievirus and adenovirus receptor (CAR) mediates atrioventricular-node function and connexin 45 localization in the murine heart. J Clin Invest. 2008;118(8): 2758-2770.

14. Anders M, Rösch T, Küster $K$, et al. Expression and function of the coxsackie and adenovirus receptor in Barrett's esophagus and associated neoplasia. Cancer Gene Ther. 2009;16(6):508-515

15. Stecker K, Vieth M, Koschel A, Wiedenmann
B, Röcken C, Anders M. Impact of the coxsackievirus and adenovirus receptor on the adenoma-carcinoma sequence of colon cancer. Br J Cancer. 2011;104(9):1426-1433.

16. Saito K, Sakaguchi M, Iioka H, et al. Coxsackie and adenovirus receptor is a critical regulator for the survival and growth of oral squamous carcinoma cells. Oncogene. 2014;33(10):1274-1286.

17. Nalbantoglu J, Pari G, Karpati G, Holland PC. Expression of the primary coxsackie and adenovirus receptor is downregulated during skeletal muscle maturation and limits the efficacy of adenovirus-mediated gene delivery to muscle cells. Hum Gene Ther. 1999;10(6):1009-1019.

18. Zheng J, Huynh H, Umikawa M, Silvany R, Zhang CC. Angiopoietin-like protein 3 supports the activity of hematopoietic stem cells in the bone marrow niche. Blood. 2011;117(2):470-479.

19. Zheng J, Umikawa M, Cui C, et al. Inhibitory receptors bind ANGPTLs and support blood stem cells and leukaemia development. Nature. 2012;485(7400):656-660.

20. Zheng J, Umikawa M, Zhang S, et al. Ex vivo expanded hematopoietic stem cells overcome the MHC barrier in allogeneic transplantation. Cell Stem Cell. 2011;9(2):119 130.

21. Kansaku A, Hirabayashi S, Mori H, et al. Ligand-of-Numb protein $\mathrm{X}$ is an endocytic scaffold for junctional adhesion molecule 4. Oncogene. 2006;25(37):5071-5084.

22. Rebel VI, Hartnett S, Denham J, Chan M, Finberg R, Sieff CA. Maturation and lineage-specific expression of the coxsackie and adenovirus receptor in hematopoietic cells. Stem Cells. 2000;18(3):176-182.

23. Dorner AA, Wegmann F, Butz S, et al. Coxsackievirus-adenovirus receptor (CAR) is essential for early embryonic cardiac development. J Cell Sci. 2005;118(15):3509-3521.

24. Grzegorzewski K, Ruscetti FW, Usui N, et al. Recombinant transforming growth factor beta 1 and beta 2 protect mice from acutely lethal doses of 5-fluorouracil and doxorubicin. J Exp Med. 1994;180(3):1047-1057.

25. Cheng C-W, Adams GB, Perin L, et al. Prolonged fasting reduces IGF-1/PKA to promote hematopoietic-stem-cell-based regeneration and reverse immunosuppression. Cell Stem Cell. 2014;14(6):810-823.

26. McGill MA, McGlade CJ. Mammalian numb proteins promote Notch1 receptor ubiquitination and degradation of the Notch1 intracellular domain. J Biol Chem. 2003;278(25): 23196-23203.

27. Bray SJ. Notch signalling: a simple pathway becomes complex. Nat Rev Mol Cell Biol. 2006;7(9):678-689.

28. Chapman G, Liu L, Sahlgren C, Dahlqvist C, Lendahl U. High levels of Notch signaling down-regulate Numb and Numblike. J Cell Biol. 2006;175(4):535-540.

29. Poulos MG, Guo P, Kofler NM, et al.
Endothelial Jagged-1 is necessary for homeostatic and regenerative hematopoiesis. Cell Rep. 2013;4(5):1022-1034.

30. Mercher T, Cornejo MG, Sears C, et al. Notch signaling specifies megakaryocyte development from hematopoietic stem cells. Cell Stem Cell. 2008;3(3):314-326.

31. Radtke F, Wilson A, Stark G, et al. Deficient $T$ cell fate specification in mice with an induced inactivation of Notch1. Immunity. 1999;10(5):547-558.

32. Moellering RE, Cornejo M, Davis TN, et al Direct inhibition of the NOTCH transcription factor complex. Nature. 2009;462(7270): 182-188.

33. Weng AP, Nam Y, Wolfe MS, et al. Growth suppression of pre-T acute lymphoblastic leukemia cells by inhibition of notch signaling. Mol Cell Biol. 2003;23(2):655-664.

34. Maillard I, Weng AP, Carpenter AC, et al. Mastermind critically regulates Notch-mediated lymphoid cell fate decisions. Blood. 2004:104(6):1696-1702.

35. Platta CS, Greenblatt DY, Kunnimalaiyaan M, Chen HJJoSR. Valproic acid induces Notch1 signaling in small cell lung cancer cells. J Surg Res. 2008;148(1):31-37.

36. Mirza M, Raschperger E, Philipson L, Pettersson RF, Sollerbrant K. The cell surface protein coxsackie-and adenovirus receptor (CAR) directly associates with the Ligand-ofNumb Protein-X2 (LNX2). Exp Cell Res. 2005;309(1):110-120.

37. Sollerbrant K, Raschperger E, Mirza M, et al. The Coxsackievirus and adenovirus receptor (CAR) forms a complex with the PDZ domain-containing protein ligand-of-numb protein-X (LNX). J Biol Chem. 2003;278(9): 7439-7444.

38. Himburg HA, Doan PL, Quarmyne M, et al. Dickkopf-1 promotes hematopoietic regeneration via direct and niche-mediated mechanisms. Nat Med. 2017;23(1):91-99.

39. Doan PL, Russell JL, Himburg HA, et al. Tie $2+$ bone marrow endothelial cells regulate hematopoietic stem cell regeneration following radiation injury. Stem Cells. 2013;31(2):327-337.

40. Hooper AT, Butler JM, Nolan DJ, et al. Engraftment and reconstitution of hematopoiesis is dependent on VEGFR2mediated regeneration of sinusoidal endothelial cells. Cell Stem Cell. 2009;4(3): 263-274.

41. Doan PL, Himburg HA, Helms K, et al. Epidermal growth factor regulates hematopoietic regeneration after radiation injury. Nat Med. 2013;19(3):295-304.

42. Himburg HA, Muramoto GG, Daher P, et al. Pleiotrophin regulates the expansion and regeneration of hematopoietic stem cells. Nat Med. 2010;16(4):475-482.

43. Pajcini K, Speck N, Pear W. Notch signaling in mammalian hematopoietic stem cells. Leukemia. 2011;25(10):1525-1532.

44. Bigas A, Espinosa L. Hematopoietic stem 
cells: to be or Notch to be. Blood. 2012;119 (14):3226-3235

45. Maillard I, Koch U, Dumortier A, et al. Canonical notch signaling is dispensable for the maintenance of adult hematopoietic stem cells. Cell Stem Cell. 2008;2(4):356366.

46. Mancini SJ, Mantei N, Dumortier A, Suter U, MacDonald HR, Radtke F. Jagged1-dependent Notch signaling is dispensable for hematopoietic stem cell self-renewal and differentiation. Blood. 2005;105(6):2340-2342.

47. Varnum-Finney B, Xu L, Brashem-Stein C, et al. Pluripotent, cytokine-dependent, hematopoietic stem cells are immortalized by constitutive Notch1 signaling. Nat Med. 2000;6(11):1278-1281.

48. Stier S, Cheng T, Dombkowski D, Carlesso $\mathrm{N}$, Scadden DT. Notch1 activation increases hematopoietic stem cell self-renewal in vivo and favors lymphoid over myeloid lineage outcome. Blood. 2002.99(7) 2369-2378.

49. Spana EP, Doe CO. Numb antagonizes Notch signaling to specify sibling neuron cell fates. Neuron. 1996;17(1):21-26.

50. Guo M, Jan LY, Jan YN. Control of daughter cell fates during asymmetric division: interaction of Numb and Notch. Neuron. 1996;17 (1):27-41.

51. Wilson A, Ardiet D-L, Saner C, et al. Normal hemopoiesis and lymphopoiesis in the combined absence of numb and numblike. J Immunol. 2007;178(11):6746-6751.

52. Wu Y-C, Lee K-S, Song Y, Gehrke S, Lu B. The bantam microRNA acts through Numb to exert cell growth control and feedback regulation of Notch in tumor-forming stem cells in the Drosophila brain. PLoS Genet. 2017;13(5):e1006785.

53. Nie J, McGill MA, Dermer M, Dho SE, Wolting CD, McGlade CJ. LNX functions as a RING type E3 ubiquitin ligase that targets the cell fate determinant Numb for ubiquitin-dependent degradation. EMBO J. 2002;21(1-2):93-102.

54. van Raaij MJ, Chouin E, van der Zandt $\mathrm{H}$ Bergelson JM, Cusack S. Dimeric structure of the coxsackievirus and adenovirus receptor D1 domain at $1.7 \AA$ resolution. Structure. 2000;8(11):1147-1155

55. Simsek T, Kocabas F, Zheng J, et al. The distinct metabolic profile of hematopoietic stem cells reflects their location in a hypoxic niche. Cell Stem Cell. 2010;7(3):380-390.

56. Spencer JA, Ferraro F, Roussakis E, et al. Direct measurement of local oxygen concentration in the bone marrow of live animals. Nature. 2014;508(7495):269-273.

57. Küster K, Koschel A, Rohwer N, Fischer A, Wiedenmann B, Anders M. Downregulation of the coxsackie and adenovirus receptor in cancer cells by hypoxia depends on HIF- $1 \alpha$. Cancer Gene Ther. 2010;17(2):141-146. 